\title{
Carbon structures and defect planes in diamond at high pressure
}

\author{
Silvana Botti, ${ }^{1}$ Maximilian Amsler, ${ }^{2}$ José A. Flores-Livas, ${ }^{1}$ Paul Ceria, ${ }^{1}$ Stefan Goedecker, ${ }^{2}$ and Miguel A. L. Marques ${ }^{1}$ \\ ${ }^{1}$ Institut Lumière Matière, UMR5306, Université Lyon 1-CNRS, Université de Lyon, F-69622 Villeurbanne Cedex,France \\ ${ }^{2}$ Department of Physics, Universität Basel,Klingelbergstrasse 82, 4056 Basel, Switzerland
}

(Received 26 October 2012; published 8 July 2013)

\begin{abstract}
We performed a systematic structural search of high-pressure carbon allotropes for unit cells containing from 6 to 24 atoms using the minima hopping method. We discovered a series of new structures that are consistently lower in enthalpy than the ones previously reported. Most of these include $(5+7)$ - or $(4+8)$-membered rings and can therefore be placed in the families proposed by H. Niu et al. [Phys. Rev. Lett. 108, 135501 (2012)]. However, we also found three more families with competitive enthalpies that contain $(5+5+8)$-membered rings, $s p^{2}$ motives, or buckled hexagons. These structures are likely to play an important role in dislocation planes and structural defects of diamond and hexagonal diamond.
\end{abstract}

DOI: 10.1103/PhysRevB.88.014102

PACS number(s): 61.66.Bi, 61.50.Ks, 62.50.-p

\section{INTRODUCTION}

Carbon has long since enticed the imagination of researchers. Not only is it the key component of life, but it also finds numerous applications in diverse fields of technology. Moreover, and above all, carbon shows a remarkable capability to form different bonds and different structures. The most stable phase of carbon, at ambient conditions, is graphite, where the atoms crystallize in hexagonal $s p^{2}$ planes bound together by weak van der Waals forces. Other bulk allotropic metastable forms found in nature include diamond and lonsdaleite (hexagonal diamond), where the carbon atoms are arranged in a $s p^{3}$ network. Even if diamond becomes very quickly the most stable form as a function of applied pressure, the barrier for the direct transformation between graphite and diamond is very high, and the conversion requires high temperatures and pressures. For this reason, the graphitic structure is stable up to pressures of $10 \mathrm{GPa}$ when compressed at low (ambient) temperature. At that point, it undergoes a phase transformation to a still-undetermined structure. ${ }^{1-6}$

Many different works, mostly theoretical but also a few experimental, have tried to identify what this unknown structure is. In fact, more than 15 low-energy structures ${ }^{7-18}$ have been put forward to explain the experimental x-ray diffraction pattern. These are $s p^{3}$ phases that were mostly obtained through $a b$ initio crystal prediction methods, where the energy landscape of carbon at high pressure is explored. In fact, we can say that carbon has become the benchmark for structural prediction methods, with several groups striving to obtain the lowest minimum using all available codes and methods, such as systematic search, ${ }^{19}$ genetic algorithms, ${ }^{20}$ particleswarm optimization, ${ }^{21}$ metadynamics, ${ }^{22}$ the minima-hopping method, ${ }^{23}$ etc. Furthermore, many of these phases have been characterized theoretically, through the calculation of their electronic structure, optical and mechanical properties, ${ }^{24}$ IR spectra, ${ }^{25}$ Raman spectra, ${ }^{26}$ etc. Nevertheless, the crystal structure of cold compressed graphite is still the subject of intense discussions in literature.

The reasons are multiple. From the experimental side, the $\mathrm{x}$-ray diffraction pattern of the novel structure does not have enough resolution for a direct experimental characterization. In addition, due to the significant broadening of the main peaks (see Fig. 5), most of the proposed theoretical structures fit the experimental data equally well. Many other measurements exist, mainly coming from Raman scattering experiments on samples under pressure. ${ }^{27}$ In this case the interpretation of the spectra is profoundly complicated by the large diamond peak coming from the anvil cell, which overlaps with the main active Raman modes of the theoretical structures. Several other peaks are found in experiments of compressed graphite, ${ }^{7}$ nanotubes, ${ }^{28}$ fullerenes, ${ }^{29}$ etc., and even in meteorites, ${ }^{30}$ but these peaks are not entirely reproducible and have found numerous different interpretations. ${ }^{31-35}$

On the other hand, from the theoretical side, the search for low-enthalpy structures is a more complicated mathematical problem than the search for the lowest-enthalpy structure. In fact it is obviously possible to obtain an infinite number of periodic arrangements with energies as close as we want (even infinitesimally close) to the ground state simply by creating large enough supercells with localized point defects. Of course, one would argue that these are not novel structures, but the distinction between a novel crystal and a structure with defects is again not well defined mathematically, as it relies on qualitative observations. Up to recently, this problem had been circumvented in theoretical works by using small unit cells that necessarily lead to substantially different arrangements of atoms.

In this paper, we go a step forward and perform an unbiased structural search of low-enthalpy phases of carbon under pressure with relatively large unit cells up to 24 atoms. In this way, we not only find unreported geometries that have a consistently lower enthalpy than previously proposed phases (for a fixed number of atoms per unit cell) but also witness the transition from what we can safely define as different allotropes of carbon to structures consisting of diamond with planes (or lines) of defects.

\section{COMPUTATIONAL DETAILS}

We used the minima-hopping method,,$^{23,36}$ an efficient crystal-structure prediction algorithm designed to predict the low-energy structures of a system given solely its chemical composition. The energy surface is explored by performing consecutive short molecular dynamics escape steps followed 
by local geometry relaxations taking into account both atomic and cell variables. The initial velocities for the molecular dynamics trajectories are chosen approximately along soft-mode directions, thus allowing efficient escapes from local minima and aiming towards low-energy structures. The predictive power of this approach has been demonstrated in a wide range of applications. ${ }^{7,37-39}$

Most often, structural prediction of crystals is performed using $a b$ initio density functional theory (DFT). This is clearly the preferred method as DFT allows the study of the whole periodic table with good accuracy. However, as the number of atoms in the unit cell (and, consequently, the number of local minima of the enthalpy surface) grows, DFT simulations become prohibitively long. Therefore, we resorted to a less demanding alternative, namely, tight-binding theory as implemented in the DFTB+ package. ${ }^{40}$ Note that tight binding is a reliable and commonly used technique for carbon materials and that the Slater-Koster parameters are well tested for both $s p^{2}$ and $s p^{3}$ forms of carbon. In this way, we managed to performed minima-hopping runs with different cells containing $4,6,8,10,12,14,16,18,20,22$, and 24 atoms in the unit cell at a pressure of $20 \mathrm{GPa}$. All runs started from a random structure and managed to obtain the diamond ground state and a large number of low-lying local minima. The symmetry of the resulting structures was obtained with FINDSYM. ${ }^{41}$

The most promising structures were then always refined at the DFT level within the local-density approximation (LDA) to the exchange-correlation functional using the code VASP. ${ }^{42}$ Calculations used Monkhorst-Pack $k$-point grids that assured convergence of the total energy to better than $0.01 \mathrm{eV}$ per atom. We notice that the energy ordering of the different structures with the LDA follows to a large extent the one of tight binding, which proves that the tight-binding results are already qualitatively good and our procedure to combine tight binding and DFT calculations is reliable.

\section{RESULTS}

Our results are summarized in Fig. 1 where we depict the enthalpies, calculated at $20 \mathrm{GPa}$, of the low-lying phases of carbon as a function of the number of atoms in the unit

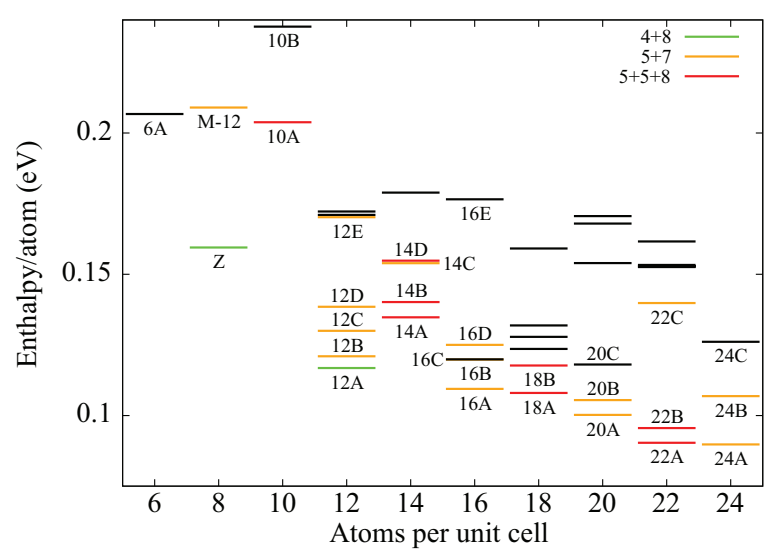

FIG. 1. (Color online) Enthalpies (at $20 \mathrm{GPa}$ ) of the most interesting structures found in this work as a function of the number of atoms in the unit cell.

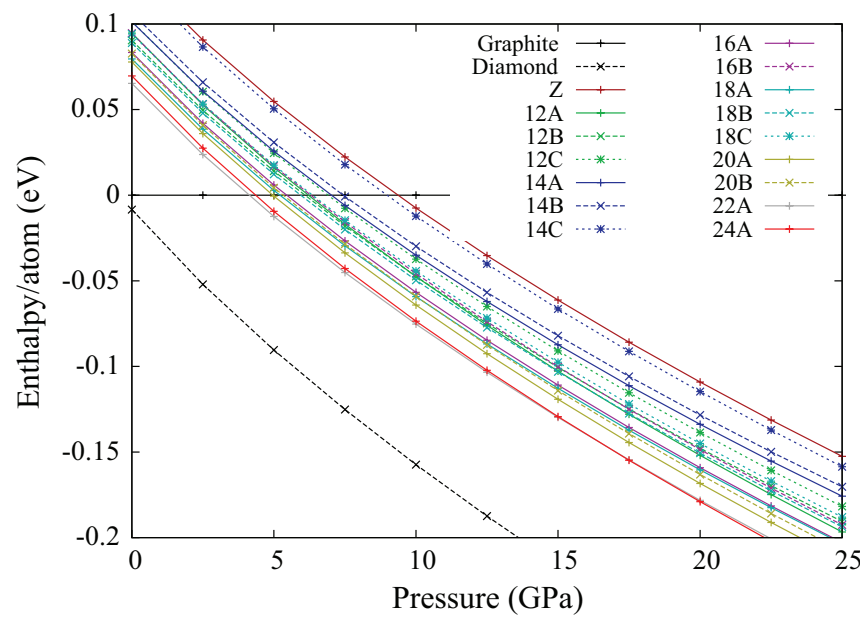

FIG. 2. (Color online) Calculated enthalpy difference per atom with respect to graphite for different structures of carbon as a function of applied pressure.

cell. The labels used to identify the new structures and the new families are also defined in Fig. 1. The crystallographic information files of the structures reported in Fig. 1 are given in the Supplemental Material. ${ }^{43}$ We note that this is a very small fraction of the total number of local minima found in the minima-hopping runs. The general trend is that the enthalpy decreases with the number of atoms in the unit cell. Some exceptions do occur, for 10 and 14 atom cells, which seem to be rather complicated numbers to build the $s p^{3}$ networks that are favored at high pressure. We furthermore emphasize that, with eight atoms, $Z$-carbon ${ }^{7}$ defiantly remains the lowest-energy structure. Our structures are consistently lower in enthalpy than the ones found in the literature and, in particular, the ones from Ref. 44.

In Fig. 2 we show the variation of the enthalpy per atom of our carbon phases with respect to graphite as a function of applied pressure. As these are mostly $s p^{3}$, it is not surprising that the overall variation of the curves follows quite closely the one of diamond. Furthermore, with very few exceptions, the curves are essentially parallel to one another in the pressure range from 0 to $20 \mathrm{GPa}$. As the enthalpies of several structures are lower than the one of $Z$-carbon, they cross the graphite line (i.e., they become more stable than graphite) for pressures below $10 \mathrm{GPa}$, with the minimum pressure around $4 \mathrm{GPa}$. Of course, the transition pressure can be made as low as we wish simply by increasing the number of atoms in the unit cell.

However, the most interesting information is obtained from looking at our minima structures, especially in view of the recent work of Niu et al., ${ }^{44}$ which reports a classification of carbon structures under pressure by analyzing their topological stacking. They distinguished two families, the $S$ family that included combinations of 5 - and 7 -membered rings $(5+7)$ and the $B$ family containing 4 - and 8 -membered rings $(4+8)$, which was, however, already characterized back in 1997 by Baughman et al. $^{45}$ Both families could also include the six-atom hexagonal rings typical of diamond. Based on this classification Baughman et al. and later Niu et al. built several carbon arrangements with lower enthalpy than the ones previously known, namely, $P$-carbon from the $S$ family and 

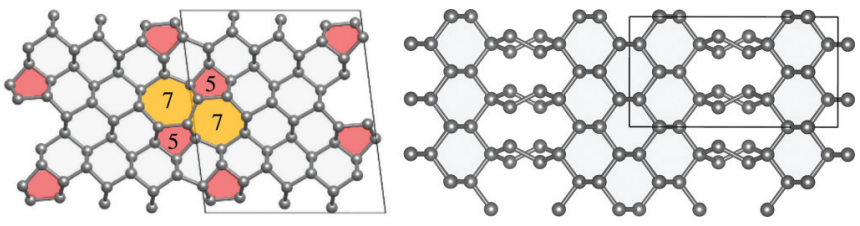

FIG. 3. (Color online) (left) Structure 24A exhibiting a $5+7$ line defect. (right) Structure 20C exhibiting a defect plane composed entirely by buckled hexagons.

$R$-carbon from the $B$ family. For clarity in the following we will depart from the convention of Niu et al. ${ }^{44}$ and label the families " $5+7$ " and " $4+8$ ".

In line with other studies with 16-atom unit cells, we find in our simulations structures consisting of "defect planes" with the previously proposed $(5+7)$-membered (such as $\mathrm{M}-12,12 \mathrm{~B}, 16 \mathrm{~A}, 20 \mathrm{~A})$ or $(4+8)$-membered rings (such as $\mathrm{Z}, 12 \mathrm{~A}$ ), interleaving several layers of diamond. However, we also find three new families, namely, one showing $(5+$ $5+8$ )-membered rings (i.e., two 5-membered rings for each 8-membered ring, such as 6B, 10A, 14A, 18A, 22A), a family that includes a few $s p^{2}$ carbons in the $s p^{3}$ arrangement (such as 10B, 16C), and another closely related family where the $s p^{2}$ hexagons become buckled (such as 20C, 22C). A representative of the $s p^{2}$ family was predicted as early as 1983 by Hoffmann et al. ${ }^{46}$ Figures 3 and 4 show illustrative examples of these families.

The appearance of the $(5+5+8)$-membered rings is perhaps less surprising, especially if we make the parallel with graphitic materials. In this case, it is well known that the most common defect stems from the $90^{\circ}$ rotation of a C-C bond leading to the creation of a pentagon and a heptagon, the so-called Stone-Wales defect. By performing a series of Stone-Wales transformations we can obtain completely flat "graphene" layers consisting of pentagons and heptagons (and hexagons), the so-called haeckelites. ${ }^{47}$ Of course, further Stone-Wales transformations can lead to octagons and so on. Analogously, for $s p^{3}$ carbon, we can imagine a similar (threedimensional) picture where one transforms the 6-membered rings of the ideal diamond lattice to the $5+7$ structure and then either to the $4+8$ or the $5+5+8$ structure. On this basis we can speculate on the existence of unit cells containing 9-membered rings. We do not think that it is likely that a $3+9$ family exists as a three-atom carbon ring would certainly lead to too much stress in the deformed $s p^{3}$ bonds and therefore to too high energy. On the other hand, a $4+5+9$ or a

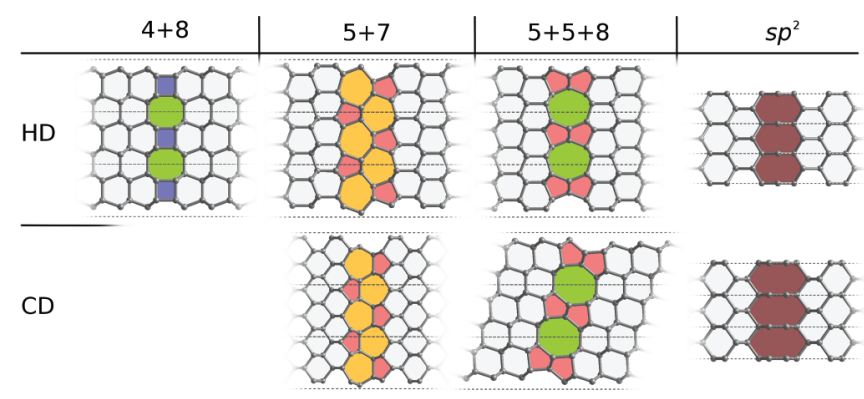

FIG. 4. (Color online) Defect planes in hexagonal and cubic diamond (HD and $\mathrm{CD}$, respectively).
$5+5+5+9$ structure cannot so easily be ruled out, despite the fact that they do not appear in our simulations. However, we notice that the existence of 9-membered rings would likely lead to large cell volumes and therefore to high enthalpies.

We can further analyze our results by looking at the lowestenthalpy structure as a function of the number of atoms in the unit cell (see Fig. 1). For more than 10 atoms in the unit cell, the minimum structure essentially alternates between $5+7$ and $5+5+8$. This can be easily understood from a geometrical basis, as one needs an extra four carbon atoms per unit cell to accommodate an extra layer of diamond in between the defect planes. Extrapolating the results to a smaller number of atoms per unit cell tells us that there must exist a structure with zero diamond layers of the $5+7$ family with 8 atoms per unit cell (it is actually the so-called M-12 carbon) and of the $5+5+8$ family with 6 atoms. This is indeed the case, and we found in our minima-hopping runs the above-mentioned phases.

If we regard the $4+8,5+7,5+5+8$, and $s p^{2}$ families as bulk diamond with defect planes, we can calculate the formation enthalpy of these planes and the interaction enthalpy. For an accurate estimation of the formation enthalpy we selected specific structures from the pool of low-lying minima for each kind of defect plane embedded in both cubic (CD) and hexagonal diamond (HD): $12 \mathrm{~B}$ and 12D for the $5+7$ family, $14 \mathrm{~A}$ and $18 \mathrm{C}$ for the $5+5+8$ family, and $16 \mathrm{C}$ and $10 \mathrm{~B}$ for the $s p^{2}$ family. For the $4+8$ family we only found $Z$ carbon polytypes which are embedded in HD. By successively increasing the perfect $\mathrm{CD}$ or HD slab between the defect layers (see Fig. 4) and by comparing the total energy/enthalpy with the perfect diamond structure of equivalent dimension the formation energy/enthalpy of the isolated defect planes was extrapolated and compiled in Table I. Strikingly, all families of defect planes, with the exception of $s p^{2}$ planes, exhibit lower formation energies if they are embedded in HD compared to CD. Furthermore, the $5+5+8$ defects in HD exhibit the lowest formation energy/enthalpy, which is in good agreement with the discovery of such structures with increasing simulation cell size during our minima hopping simulations.

We now look at our results for the 24-atom cells. At this size of the simulation cell, instead of finding a structure belonging to the $5+5+8$ planar (2D) defect family as the lowest enthalpy structure we observe the formation of low-lying defect lines (1D; see Fig. 3). These are made of two $(5+7)$-membered rings surrounded by layers of diamond in the $x$ and $y$ directions. Of course, we can also expect the appearance of cells containing (OD) point defects with

TABLE I. Formation energies $\Delta E_{\sigma}$ and enthalpies $\Delta H_{\sigma}$ at $20 \mathrm{GPa}$ per unit area (in $\mathrm{meV} / \AA^{2}$ ) for various defect planes embedded in cubic (CD) and hexagonal diamond (HD). Here n.a. indicates not applicable.

\begin{tabular}{lcccc}
\hline \hline Defect type & $\mathrm{CD} \Delta E_{\sigma}$ & $\mathrm{CD} \Delta H_{\sigma}$ & $\mathrm{HD} \Delta E_{\sigma}$ & $\mathrm{HD} \Delta H_{\sigma}$ \\
\hline $4+8$ & n.a. & n.a. & 194.7 & 218.9 \\
$5+7$ & 297.2 & 307.5 & 151.1 & 172.9 \\
$5+5+8$ & 302.0 & 365.8 & 136.4 & 167.1 \\
$s p^{2}$ & 361.6 & 420.8 & 531.7 & 619.7 \\
\hline \hline
\end{tabular}




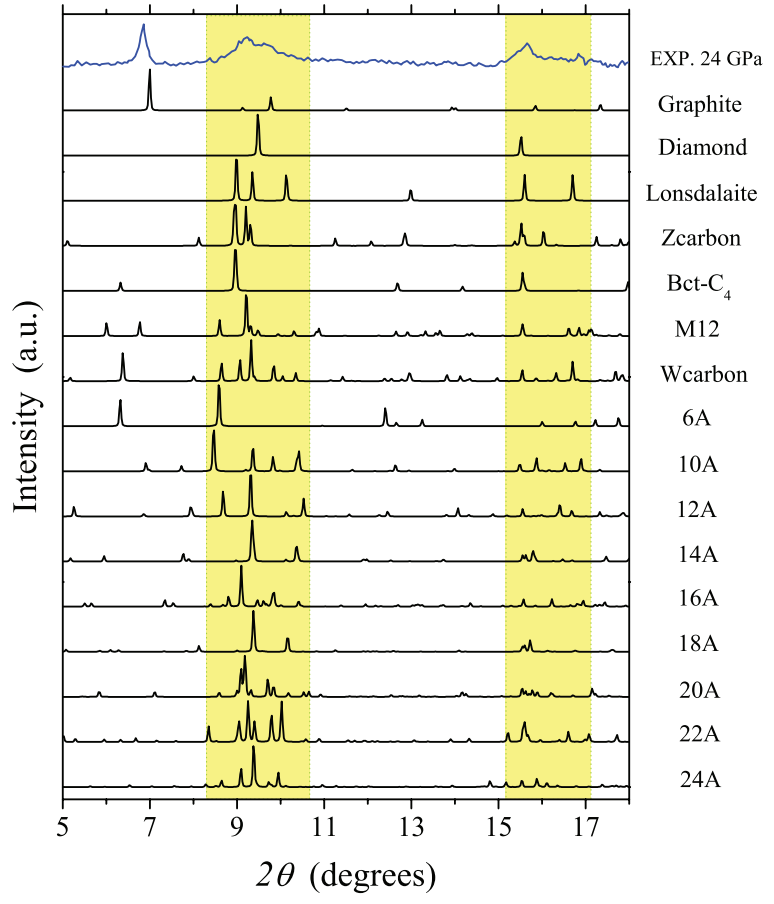

FIG. 5. (Color online) X-ray diffraction patterns of carbon polytypes under pressure. The blue line (top) represents the acquired experimental spectrum of compressed graphite at $24 \mathrm{GPa}$ from Ref. 6. Black lines show the simulated spectra $(\lambda=0.3329 \AA)$ of different structures of carbon at $20 \mathrm{GPa}$. The yellow areas serve as a guide for the eye to show the principal changes found in the experimental diffraction.

similar geometries, but this will require larger unit cells that we estimate can have 75-100 atoms.

Finally, in Fig. 5 we show the simulated diffraction patterns for selected carbon allotropes compared to the experimental spectrum of Ref. 6 . The diffraction curves of the new structures are arranged following their order of appearance in Fig. 2 and system cell size. In the experimental spectra one can distinguish the two principal reflections related to the $s p^{3}$ bonding of carbon atoms. The first contribution can be found between $8.5^{\circ}$ and $10.5^{\circ}$ (first yellow shaded area) and the second contribution is between $15.2^{\circ}$ and $17^{\circ}$ (second yellow shaded area). Like most of the carbon allotropes previously proposed in literature, the whole series of new structures presented here matches well the broad experimental peaks.

We remark that at room temperature kinetics will not allow ordering the defects; therefore actual samples will contain a mixture of several semidegenerate defect phases. Locally, however, the motives presented here should be, in principle, detectable.

\section{SUMMARY AND CONCLUSIONS}

We presented a systematic study of the low-enthalpy structures of carbon under pressure using unit cells with 6 to 24 atoms. The structures can be classified in 5 different families, characterized by planes having layers with $s p^{2}$ carbons or $(4+8)-,(5+7)-$, or $(5+5+8)$-membered rings. For cells having between 10 and 22 atoms, the minimumenthalpy structures essentially alternate between the $5+7$ and the $5+5+8$ families. Furthermore, we were able to estimate the energy of the isolated defect planes. Finally, for 24-atom cells we observed the appearance of unit cells consisting of diamond networks including $5+7$ one-dimensional lines of defects. We believe that the motives studied here are important in the geometry of grain boundaries or dislocation planes and can play an important role in the low-energy spectrum of line and point defects of diamond.

\section{ACKNOWLEDGMENTS}

Financial support provided by the Swiss National Science Foundation is gratefully acknowledged. M.A.L.M. acknowledges support from the French ANR (Grant No. ANR-08CEXC8-008-01). Computational resources were provided by the Swiss National Supercomputing Center (CSCS) in Lugano and GENCI (project x2011096017) in France.
${ }^{1}$ F. P. Bundy, J. Chem. Phys. 46, 3437 (1967).

${ }^{2}$ A. F. Goncharov, I. N. Makarenko, and S. M. Stishov, Sov. Phys. JETP 69, 380 (1989).

${ }^{3}$ Y. X. Zhao and I. L. Spain, Phys. Rev. B 40, 993 (1989).

${ }^{4}$ W. Utsumi and T. Yagi, Science 252, 1542 (1991).

${ }^{5}$ E. D. Miller, D. C. Nesting, and J. V. Badding, Chem. Mater. 9, 18 (1997).

${ }^{6}$ W. L. Mao, H. kwang Mao, P. J. Eng, T. P. Trainor, M. Newville, C. chang Kao, D. L. Heinz, J. Shu, Y. Meng, and R. J. Hemley, Science 302, 425 (2003).

${ }^{7}$ M. Amsler, J. A. Flores-Livas, L. Lehtovaara, F. Balima, S. A. Ghasemi, D. Machon, S. Pailhès, A. Willand, D. Caliste, S. Botti, A. San Miguel, S. Goedecker, and M. A. L. Marques, Phys. Rev. Lett. 108, 065501 (2012).

${ }^{8}$ F. J. Ribeiro, S. G. Louie, M. L. Cohen, and P. Tangney, Phys. Rev. B 72, 214109 (2005).
${ }^{9}$ Q. Li, Y. Ma, A. R. Oganov, H. Wang, H. Wang, Y. Xu, T. Cui, H. K. Mao, and G. Zou, Phys. Rev. Lett. 102, 175506 (2009).

${ }^{10}$ K. Umemoto, R. M. Wentzcovitch, S. Saito, and T. Miyake, Phys. Rev. Lett. 104, 125504 (2010).

${ }^{11}$ X.-F. Zhou, G.-R. Qian, X. Dong, L. Zhang, Y. Tian, and H.-T. Wang, Phys. Rev. B 82, 134126 (2010).

${ }^{12}$ J. T. Wang, C. Chen, and Y. Kawazoe, Phys. Rev. Lett. 106, 075501 (2011).

${ }^{13}$ Q. Zhu, Q. Zeng, and A. R. Oganov, Phys. Rev. B 85, 201407 (2012).

${ }^{14}$ C. He, L. Sun, C. Zhang, X. Peng, K. Zhang, and J. Zhong, Solid State Commun. 152, 1560 (2012).

${ }^{15}$ J.-T. Wang, C. Chen, and Y. Kawazoe, Phys. Rev. B 85, 033410 (2012).

${ }^{16}$ F. Tian, X. Dong, Z. Zhao, J. He, and H. Wang, J. Phys. Condens. Matter 24, 165504 (2012). 
${ }^{17}$ D. Li, K. Bao, F. Tian, A. Zeng, Z. He, B. Liu, and T. Cui, Phys. Chem. Chem. Phys. 14, 4347 (2012).

${ }^{18}$ R. Zhou and X. Zeng, J. Am. Chem. Soc. 134(17), 7530 (2012).

${ }^{19}$ R. T. Strong, C. J. Pickard, V. Milman, G. Thimm, and B. Winkler, Phys. Rev. B 70, 045101 (2004).

${ }^{20}$ C. W. Glass, A. Oganov, and N. Hansen, Comput. Phys. Commun. 175, 713 (2006).

${ }^{21}$ Y. Wang, J. Lv, L. Zhu, and Y. Ma, Phys. Rev. B 82, 094116 (2010).

${ }^{22}$ A. Laio and M. Parrinello, Proc. Natl. Acad. Sci. USA 99, 12562 (2002).

${ }^{23}$ S. Goedecker, J. Chem. Phys. 120, 9911 (2004).

${ }^{24}$ H. Niu, P. Wei, Y. Sun, X. Chen, C. Franchini, D. Li, and Y. Li, Appl. Phys. Lett. 99, 031901 (2011).

${ }^{25}$ Z. Li, F. Gao, and Z. Xu, Phys. Rev. B 85, 144115 (2012).

${ }^{26}$ J. A. Flores-Livas, L. Lehtovaara, M. Amsler, S. Goedecker, S. Pailhès, S. Botti, A. San Miguel, and M. A. L. Marques, Phys. Rev. B 85, 155428 (2012).

${ }^{27}$ M. Hanfland, H. Beister, and K. Syassen, Phys. Rev. B 39, 12598 (1989).

${ }^{28}$ Z. Wang, Y. Zhao, K. Tait, X. Liao, D. Schiferl, C. Zha, R. Downs, J. Qian, Y. Zhu, and T. Shen, Proc. Natl. Acad. Sci. USA 101, 13699 (2004).

${ }^{29}$ M. Álvarez-Murga, P. Bleuet, G. Garbarino, A. Salamat, M. Mezouar, and J. L. Hodeau, Phys. Rev. Lett. 109, 025502 (2012).

${ }^{30}$ D. Smith and G. Godard, Spectrochim. Acta, Part A 73, 428 (2009).

${ }^{31}$ Y. Wang, J. E. Panzik, B. Kiefer, and K. K. M. Lee, Sci. Rep. 2, 520 (2012).

${ }^{32}$ B. A. Fairchild, S. Rubanov, D. W. M. Lau, M. Robinson, I. SuarezMartinez, N. Marks, A. D. Greentree, D. McCulloch, and S. Prawer, Adv. Mater. 24, 2024 (2012).

${ }^{33}$ Y. Lin, L. Zhang, H.-K. Mao, P. Chow, Y. Xiao, M. Baldini, J. Shu, and W. L. Mao, Phys. Rev. Lett. 107, 175504 (2011).
${ }^{34}$ A. Ferrari and J. Robertson, Philos. Trans. R. Soc. London, Ser. A 362, 247 (2006).

${ }^{35}$ T. T. Irifune, A. Kurio, S. Sakamoto, T. Inoue, and H. Sumiya, Nature (London) 421, 599 (2003).

${ }^{36}$ M. Amsler and S. Goedecker, J. Chem. Phys. 133, 224104 (2010).

${ }^{37}$ K. Bao, S. Goedecker, K. Koga, F. Lançon, and A. Neelov, Phys. Rev. B 79, 041405 (2009).

${ }^{38}$ J. A. Flores-Livas, M. Amsler, T. J. Lenosky, L. Lehtovaara, S. Botti, M. A. L. Marques, and S. Goedecker, Phys. Rev. Lett. 108, 117004 (2012).

${ }^{39}$ M. Amsler, J. A. Flores-Livas, T. D. Huan, S. Botti, M. A. L. Marques, and S. Goedecker, Phys. Rev. Lett. 108, 205505 (2012).

${ }^{40}$ B. Aradi, B. Hourahine, and T. Frauenheim, J. Phys. Chem. A 111, 5678 (2007).

${ }^{41}$ H. T. Stokes and D. M. Hatch, J. Appl. Crystallogr. 38, 237 (2005).

${ }^{42}$ G. Kresse and J. Furthmüller, Comput. Mater. Sci. 6, 15 (1996).

${ }^{43}$ See Supplemental Material at http://link.aps.org/supplemental/ 10.1103/PhysRevB.88.014102 for the crystallographic information files of the low-enthalpy structures reported in this paper. All the structures, together with symmetry analysis, can be also found at http://www.tddft.org/bmg/physics/structures/C/.

${ }^{44}$ H. Niu, X.-Q. Chen, S. Wang, D. Li, W. L. Mao, and Y. Li, Phys. Rev. Lett. 108, 135501 (2012).

${ }^{45}$ R. H. Baughman, A. Y. Liu, C. Cui, and P. J. Schields, Synth. Met. 86, 2371 (1997).

${ }^{46}$ R. Hoffmann, T. Hughbanks, M. Kertesz, and P. H. Bird, J. Am. Chem. Soc. 105, 4831 (1983).

${ }^{47}$ H. Terrones, M. Terrones, E. Hernández, N. Grobert, J.-C. Charlier, and P. M. Ajayan, Phys. Rev. Lett. 84, 1716 (2000). 